\title{
Cerebral Small Vessel Disease in Pseudoxanthoma Elasticum: Three Cases
}

\author{
Aleksandra M. Pavlovic, Jasna Zidverc-Trajkovic, Marija M. Milovic, \\ Dragan M. Pavlovic, Zagorka Jovanovic, Milija Mijajlovic, Mirjana Petrovic, \\ Vladimir S. Kostic, Nada Sternic
}

\begin{abstract}
Background: Cerebral small vessel disease is rarely described in association with pseudoxanthoma elasticum (PXE), a hereditary connective tissue disorder with skin, eye and vascular manifestations. This autosomally inherited elastic tissue disease has been attributed to mutations in the ABCC6 gene located on chromosome 16p13.1. Different stroke mechanisms are suggested in PXE patients, arterial hypertension and accelerated atherosclerosis being the leading ones. Case Descriptions: Case 1: A 49-year-old man with history of mild hypertension presented with recurrent transient ischemic attacks. At the age of 42 , evaluation for progressive visual loss and skin changes led to diagnosis of PXE. Brain magnetic resonance imaging (MRI) disclosed multiple lacunar infarctions and confluent periventricular white matter lesions (WML). Case 2: A 71-year-old woman with history of mild hypertension suffered rightsided stroke. Diagnosis of PXE was made at the age of 48 due to severe visual loss and skin changes. Brain MRI revealed multiple lacunar infarctions and subcortical ischemic leukoencephalopathy. Case 3: A 47-year-old woman with prominent skin changes and bilateral amblyopia developed right-sided weakness. Skin biopsy confirmed PXE. Several lacunar infarcts in deep white matter and pons were revealed on MRI. Discussion: We present three patients with clinical and histopathological features of PXE who presented with multiple lacunar strokes, two with extensive confluent WML. These cases illustrate that PXE is a rare but significant risk factor for small vessel disease and stroke in patients of all age groups. Occlusive small vessel disease and subsequent lacunar infarcts and WML represent important PXE manifestations.
\end{abstract}

RÉSUMÉ: Maladie des petits vaisseaux cérébraux dans le pseudo xanthome élastique : à propos de trois observations. Introduction: Une maladie des petits vaisseaux cérébraux associée au pseudo xanthome élastique (PXÉ), une maladie héréditaire autosomique du tissus conjonctif impliquant la peau, les yeux et les vaisseaux a rarement été décrite. Elle est due à une mutation du gène ABCC6 qui est situé sur le chromosome 16p13,1. On a suggéré que plusieurs mécanismes différents pouvaient être en cause chez les patients qui ont un PXÉ et des accidents vasculaires cérébraux, dont l'hypertension artérielle et une athérosclérose accélérée. Observations: Premier cas: Il s'agit d'un homme âgé de 49 ans ayant une histoire d'hypertension légère, qui a consulté pour des accidents ischémiques transitoires. Un diagnostic de PXÉ a été posé à l'âge de 42 ans suite à une évaluation motivée par une perte de vision progressive et des changements au niveau de la peau. L'imagerie par résonance magnétique (IRM) a montré la présence de multiples infarctus lacunaires et de lésions confluentes de la substance blanche périventriculaire (LSB). Deuxième cas: Une femme âgée de 71 ans ayant une histoire d'hypertension légère, a subi un accident vasculaire cérébral droit. Un diagnostic de PXÉ avait été posé chez elle à l'âge de 48 ans suite à une perte de vision sévère et à des anomalies de la peau. L'IRM cérébrale a montré de multiples infarctus lacunaires et une leukoencéphalopathie ischémique sous-corticale. Troisième cas: Une femme âgée de 47 ans ayant des anomalies importantes de la peau et une amblyopie bilatérale a présenté une faiblesse du côté droit. Le diagnostic de PXÉ a été confirmé par une biopsie cutanée. L'IRM a montré la présence de plusieurs infarctus lacunaires dans la substance blanche profonde et le pons. Discussion: Nous présentons les cas de trois patients ayant des manifestations cliniques et anatomopathologiques du PXÉ qui ont consulté pour de multiples accidents vasculaires cérébraux, dont deux étaient porteurs de LSB confluentes importantes. Ces cas illustrent que le PXÉ est un facteur de risque rare mais important de maladie des petits vaisseaux et d'accident vasculaire cérébral chez les patients de tous âges. La maladie occlusive des petits vaisseaux, les infarctus lacunaires et les LSB qui s'en suivent sont des manifestations importantes du PXÉ.

Can. J. Neurol. Sci. 2005; 32: 115-118

Cerebral small vessel disease affects small penetrating terminal arterioles supplying deep white matter and basal ganglia, and can lead to both lacunar infarcts and diffuse white matter lesions (WML). Hypertension, diabetes mellitus and atherosclerosis are considered the main risk factors for small vessel disease, but genetic factors have been implied as well. ${ }^{1}$ Pseudoxanthoma elasticum (PXE), known also as GrönbladStrandberg syndrome, is a rare heritable systemic connective tissue disorder, characterized by progressive proteoglycan accumulation and fragmented, swollen, and calcified elastic fibers in connective tissues. ${ }^{2}$ This autosomally inherited elastic

From the Institute of Neurology, (AMP, JZ-T, DMP, ZJ, MM, VSK, NS), Institute of Ophthalmology, (MMM), Clinical Center of Serbia, Belgrade, Serbia; Department of Neurology, Clinical Center Kragujevac (MP), Kragujevac, Serbia. ReCEIVED November 3, 2003. ACCEPTEDINFINALFORM OCtOBer 7, 2004. Reprint requests to: Aleksandra M. Pavlovic, Institute of Neurology, Dr. Subotica 6, 11000 Belgrade, Serbia 
tissue disorder has been attributed to mutations in the ABCC6 gene located on chromosome 16p13.1, with dominant, recessive and sporadic forms. ${ }^{3,4}$ The ABCC6 gene encodes the multidrugresistance protein 6 (MRP6), a member of the ATP-binding cassette family of proteins. It has been suggested that MRP6 is a transmembrane transporter, which normal function may be crucial for extracellular matrix deposition or turnover of connective tissue at specific sites in the body. ${ }^{5}$

There is 3.6-fold increase of stroke risk in PXE patients. ${ }^{6}$ Exclusive involvement of cerebral small vessel and WML in PXE has been described before ${ }^{6-8}$ but these cases are rarely reported. Stroke cases have never been reported in Serbian PXE population before. We describe three patients with typical skin and ocular PXE features presenting with cerebral small vessel disease and strokes.

\section{CASE RePorts}

\section{Case 1}

A 49-year-old man was admitted to our department after repeated transient ischemic attacks (TIAs) with recurrent right-sided weakness, paresthesias and dysarthria. Each episode lasted up to 30 minutes. Mild oscillatory arterial hypertension with maximal values of $160 / 100 \mathrm{mmHg}$ had been registered two months earlier. At the age of 42 evaluation for progressive amblyopia and skin changes had led to the diagnosis of PXE confirmed by skin biopsy. Family history was unremarkable. On admission, mild right hemiparesis and yellow papules and hyperelastosis of the face and neck skin were noted. Fundoscopy disclosed pronounced degenerative retinal changes with striae angioides and macular scars. Neuropsychological evaluation showed normal findings. Plasma lipid profile, fasting glucose level, oral glucose tolerance test (OGTT), fibrinogen, hematocrit and erythrocyte sedimentation rate (ESR) were normal. Electroencephalography, serum immunology, coagulation tests,

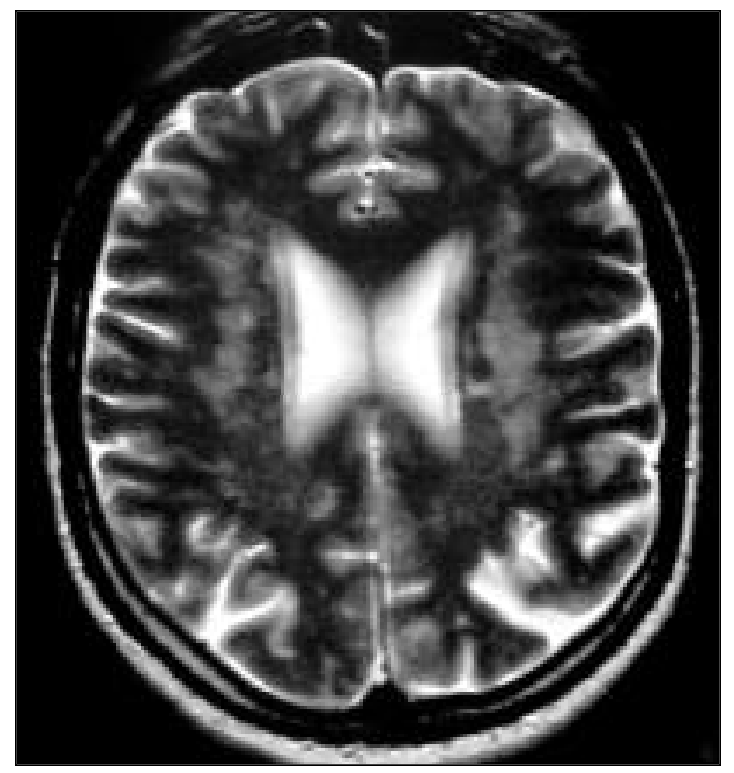

Figure 1: MRI T2-weighted: cerebral white matter lesions - Case 1 cerebrospinal fluid cytobiochemical and immunological examinations, cardiological evaluation with transthoracic echocardiography revealed normal results. Carotid duplex ultrasonography showed 50\% stenosis of the right internal carotid artery with fibrous plaques on both bifurcations. Brain MRI revealed multiple lacunar infarctions in frontal, parietal, and occipital subcortical white matter bilaterally, in left thalamus and pons, with severe confluent periventricular white matter changes and mild cortical and subcortical cerebral atrophy (Figure 1). Cerebral magnetic resonance angiography (MRA) was unremarkable. The patient was given a small dose of Aspirin (50 mg daily) and, during two-year followup, TIAs did not reoccur.

\section{Case 2}

A 71-year-old woman with history of mild oscillatory hypertension with maximal values of $140 / 100 \mathrm{mmHg}$ and stabile angina pectoris suffered acute right-sided hemiparesis and mild dysarthria. On admission neurological examination revealed bilateral amblyopia, rightsided hemiparesis with wide-based gait and mild dysarthria. Yellow papules on loose skin of the neck and axillae were observed. Her progressively poor vision suddenly deteriorated further at the age of 48 after a car accident, when ophthalmological and dermatological examinations, including skin biopsy, confirmed diagnosis of PXE. Family history for PXE and stroke was negative. Routine blood analyses disclosed elevated levels of total cholesterol $(7.84 \mathrm{mmol} / \mathrm{L} ; 303.17$ $\mathrm{mg} / \mathrm{dL}$ ) and LDL cholesterol (5.36 mmol/L; $207.27 \mathrm{mg} / \mathrm{dL}$ ). Fasting glucose level, OGTT, fibrinogen, hematocrit, ESR, serum immunology, coagulation tests and cardiological examination with echocardiography were normal. Carotid duplex ultrasonography showed intima-media thickness of $1.4 \mathrm{~mm}$ and diffuse small fibrous plaques. Neuropsychological testing showed normal finding. Cerebral MRI revealed multiple subcortical lacunar infarctions with subcortical ischemic leukoencephalopathy and moderate cortical and subcortical atrophy (Figure 2). Brain MRA was normal. The patient recovered completely. One year later she complained of progressive memory

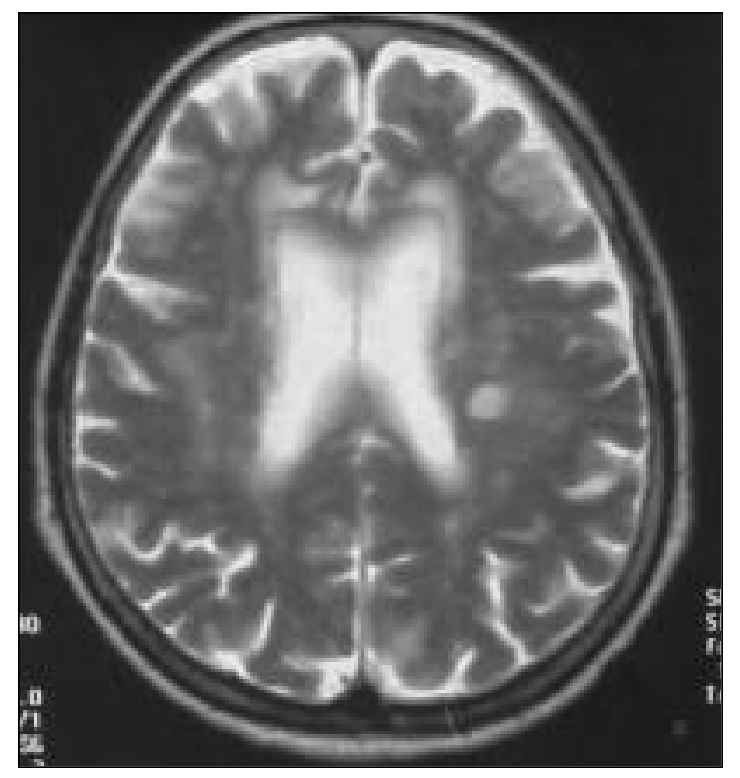

Figure 2: MRI T2-weighted: cerebral white matter lesions - Case 2 


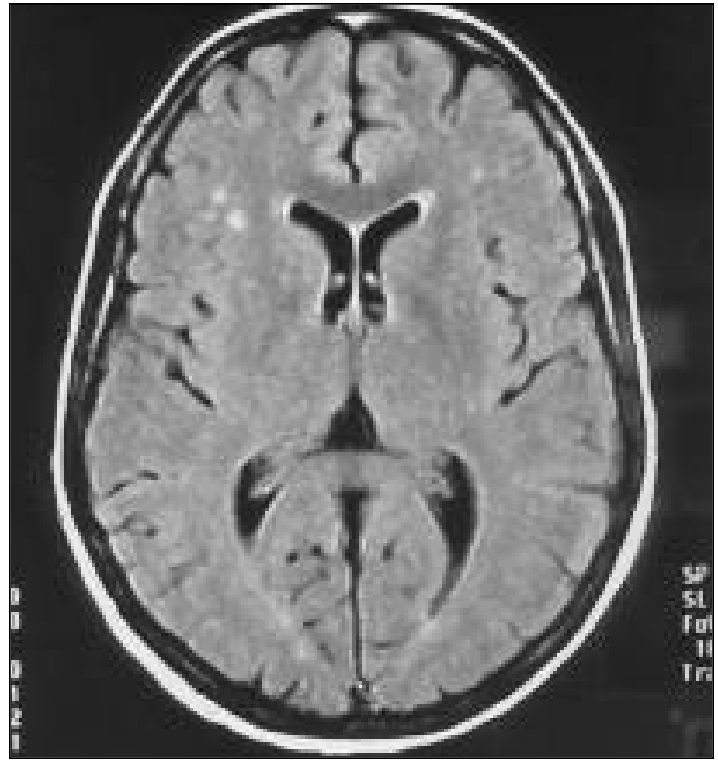

Figure 3: MRI fluid-attenuated inversion recovery (FLAIR) image: cerebral white matter lesions - Case 3

difficulties. Neuropsychological testing was repeated and there was a significant decline in delayed verbal recall on Ray Auditory Verbal Learning Test. ${ }^{9}$ Diagnosis of vascular cognitive impairment was made and nicergoline $30 \mathrm{mg}$ b.i.d. was administered.

\section{Case 3}

A 47-year-old woman presented with sudden onset of right-side hemiparesis and hemihypaesthesia that resolved in 24 hours. On examination, loose skin at the sides of the neck and in the flexural areas of the body was noted, as well as severe amblyopia. Neurological assessment revealed only right limbs hyperreflexia. Dermatological and ophthamological examination, supported by skin biopsy and fluorescein angiography, confirmed the diagnosis of PXE. There was a mild hypertension (maximal values of $150 / 100 \mathrm{mmHg}$ ) and history of 30 years smoking of 20 cigarettes per day on average, but other stroke risk factors were lacking. The patient's mother, sister and son had similar skin changes, and the mother had progressive vision loss; however, they were unavailable for evaluation. Plasma fasting glucose, OGTT, lipid profile, fibrinogen, hematocrit, ESR, as well as serum immunology, coagulation tests, cerebrospinal fluid cytobiochemical and immunological examination, cardiological assessment with echocardiography and neuropsychological testing showed normal findings. Carotid duplex ultrasonography revealed diffuse atherosclerosis. Brain MRI disclosed several lacunar infarcts in frontal deep white matter bilaterally and in the left pons (Figure 3). Cerebral MRAwas normal.

\section{Discussion}

We report three patients with PXE, a rare disease with estimated prevalence in the general population of $1: 70,000$ to 1:100,000 live births. ${ }^{3}$ Patients with PXE present with multiple systemic manifestations, including typical skin, ocular and vascular changes. All of our patients had typical PXE clinical features, which was confirmed by skin biopsy and pathohistological analysis. Unfortunately, we were not able to perform the genetic analysis.

The most important vascular PXE complication is accelerated arteriosclerosis occuring mostly in small arteries.,5,8 Usual cardiovascular manifestations include premature ischemic heart disease, renovascular hypertension and atherosclerotic peripheral vascular disease. ${ }^{5}$ These PXE manifestations, that also increase the risk of stroke, were detected in our patients as well.

Our patients presented with TIAs and strokes. Cerebrovascular lesions in PXE are not frequently described. ${ }^{10}$ Strokes in PXE occur at an adult age with single or multiple vessels occlusions or intracranial and subarachnoid hemorrhage. Cerebral small vessel involvement in PXE can result in subcortical infarcts and leukoaraiosis. The relative risk of ischemic stroke due to small-vessel disease in PXE patients under 65 years is estimated to be 3.6 compared with the general population. ${ }^{6}$ Intracranial aneurysms are infrequent in $\mathrm{PXE},{ }^{5}$ and we did not find them in our patients either.

White matter changes are rarely very extensive, ${ }^{7}$ as it was found in our Cases 1 and 2. Hirano and colleagues ${ }^{8}$ reported three PXE patients with multiple lacunar strokes and cerebral angiography excluding large vessel involvement. Multiple silent lacunar infarcts were evident on MRI examinations in all our patients. Silent strokes in PXE are probably not rare but are underdiagnosed. Mayer and co-workers ${ }^{7}$ described two women with PXE, hypertension, strokes and progressive cognitive deterioration with lacunar infarcts and diffuse WML. Compared to these cases, our patients had relatively mild clinical course, with good functional recovery and normal baseline cognitive status, in spite of extensive WML. Patient 2, however, showed signs of progressive cognitive decline consistent with vascular cognitive impairment after one-year follow-up. Patients 1 and 2 had negative family history, probably representing sporadic cases, which are often seen in PXE. ${ }^{5}$ Family occurance of PXE could not be confirmed in Patient 3 because relatives were not available for examination. The association of PXE and some particular race has not been established yet, but two of our patients were of Gypsy origin (Cases 1 and 3).

None of our patients had gastrointestinal bleeding, which can affect up to $15 \%$ of cases. Since this PXE manifestation can be life threatening, use of antithrombotic drugs in this setting is an important dilemma. ${ }^{6,11}$ We decided to give a small dose of Aspirin (50 mg daily) to Patient 1 because of recurrent TIAs, and strict control of other stroke risk factors was aimed at in all patients.

We considered nicergoline $30 \mathrm{mg}$ b.i.d. a good choice for Patient 2, since it attenuated the cognitive deterioration in elderly hypertensive patients with WML in a 24-month double-blind placebo-controlled multicentric pilot study. ${ }^{12}$ The Cochrane review ${ }^{13}$ of 14 nicergoline studies showed some, although not clear, evidence of positive effects of nicergoline in older patients with mild to moderate cognitive impairment including chronic cerebrovascular disorders. Our patient did not have any side effects during six months of follow-up and was cognitively stable.

The pathogenesis of vascular lesions in PXE is still not completely elucidated, and is probably multifactorial. 
Accelerated arteriolosclerosis and hypertension from an early age are the most important factors acting jointly. Patients with beta-thalassemia, sickle-cell disease and sickle-thalassemia may have similar histopathological and clinical manifestations, including strokes and intracranial hemorrhage, but without ABCC6 mutation. ${ }^{11,14}$

The diagnosis of PXE can be difficult. A consensus PXE meeting criteria for PXE diagnosis was formulated in $1992,{ }^{15}$ but since cardiovascular manifestations were not included nor are the sensitivity and specifity of the clinical signs clearly determined, new criteria are needed. ${ }^{5}$ Nevertheless, our patients met presently defined clinical criteria.

Although PXE is a rare disorder, and small vessel disease in PXE cases is seldom described, three patients came to our attention within a two-year period. There is a considerable variation in disease phenotype and inheritance pattern. It can be presumed that PXE occurrence in the general population is underestimated, possibly in WML patients as well, regardless of age and risk factor profile. We suggest that neurologists should consider PXE as an etiological possibility in both younger and older patients with recurrent TIAs, stroke and progressive neurological and vascular cognitive impairment, especially with cerebral small vessel involvement. These are the first PXE patients with stroke and WML reported in Serbia.

\section{ACKNOWLEDGEMENTS}

This work was supported in part by the Ministry of Science, Technologies and Development of Republic of Serbia as part of scientific project 1979.

\section{REFERENCES}

1. Schmidt R, Fazekas F, Enzinger C, et al. Risk factors and progression of small vessel disease-related cerebral abnormalities. J Neural Transm Suppl 2002; 62: 47-52.

2. Boutouyrie P, Germain DP, Tropeano A-I, et al. Compressibility of the carotid artery in patients with pseudoxanthoma elasticum. Hypertension 2001; 38: 1181-1184.

3. Struk B, Neldner KH, Rao VS, St Jean P, Lindpaintner K. Mapping of both autosomal recessive and dominant variants of pseudoxanthoma elasticum to chromosome 16p13.1. Hum Mol Genet 1997; 6: 1823-1828.

4. Bergen AA, Plomp AS, Schuurman EJ, et al. Mutations in ABCC6 cause pseudoxanthoma elasticum. Nat Genet 2000; 25: 228-231.

5. Hu X, Plomp AS, van Soest S, et al. Pseudoxanthoma elasticum: a clinical, histopathological, and molecular update. Surv Ophthalmol 2003; 48: 424-438.

6. van den Berg JS, Hennekam RC, Cruysberg JR, et al. Prevalence of symptomatic intracranial aneurysm and ischaemic stroke in pseudoxanthoma elasticum. Cerebrovasc Dis 2000; 10: 315-319.

7. Mayer SA, Tatemichi TK, Spitz JL, et al. Recurrent ischemic events and diffuse white matter disease in patients with pseudoxanthoma elasticum. Cererbovasc Dis 1994; 4: 294-297.

8. Hirano T, Hashimoto Y, Kimura K, Uchino M. Lacunar brain infarction in patients with pseudoxanthoma elasticum. Rinsho Shinkeigaku 1996; 36: 633-639.

9. Rey A. L'examen clinique en psychologie. Paris: Presses Universitaires de France, 1964.

10. Schievink WI, Michels VV, Piepgras DG. Neurovascular manifestations of heritable connective tissue disorders. A review. Stroke 1994; 25: 889-903.

11. Aessopos A, Farmakis D, Karagiorga M, Rombos I, Loucopoulos D. Pseudoxanthoma elasticum lesions and cardiac complications as contributing factors for stroke in beta-thalassemia patients. Stroke 1997; 28: 2421-2424.

12. Bes A, Orgogozo JM, Poncet M, et al. A 24-month, double-blind, placebo-controlled multicentre pilot study of the efficacy and safety of nicergoline $60 \mathrm{mg}$ per day in elderly hypertensive patients with leukoaraiosis. Eur J Neurol 1999; 6: 313-322.

13. Fioravanti M, Flicker L. Efficacy of nicergoline in dementia and other age associated forms of cognitive impairment. Cochrane Database Syst Rev 2001; 4: CD003159.

14. Aessopos A, Farmakis D, Loucopoulos D. Elastic tissue abnormalities resembling pseudoxanthoma elasticum in thalassemia and the sickling syndromes. Blood 2002; 99: 30-35.

15. Lebwohl M, Neldner K, Pope FM, et al. Classification of pseudoxanthoma elasticum: report of a consensus conference. $\mathbf{J}$ Am Acad Dermatol 1994; 30: 103-107. 